\title{
Studying and Applying Channeling at Extremely High Bunch Charges
}

\author{
Richard A. Carrigan, Jr. \\ Fermi National Accelerator Laboratory \\ Batavia, IL 60510, USA \\ Carrigan@fnal.gov \\ FERMILAB-CONF-04-529-AD
}

PACS numbers: $52.38 . \mathrm{Hb} ; 52.40 . \mathrm{Mj} ; 61.80 \mathrm{Cb} ; 61.85+\mathrm{p}$

\begin{abstract}
The potentially high plasma densities possible in solids might produce extremely high acceleration gradients. However solid-state plasmas could pose daunting challenges. Crystal channeling has been suggested as a mechanism to ameliorate these problems. A high-density plasma in a crystal lattice could quench the channeling process. There is no experimental or theoretical guidance on channeling for intense charged particle beams. An experiment has been carried out at the Fermilab A0 photoinjector to observe electron channeling radiation at high bunch charges. An electron beam with up to $8 \mathrm{nC}$ per electron bunch was used to investigate the electron-crystal interaction. No evidence was found of quenching of channeling at charge densities two orders of magnitude larger than in earlier experiments. Possible new channeling experiments are discussed for the much higher bunch charge densities and shorter times required to probe channeling breakdown and plasma behavior.
\end{abstract}

\section{Introduction}

For many years crystal channeling researchers have dreamed of using aligned crystals to accelerate charged particles. Robert Hofstadter ${ }^{1}$ mused on the limitations of conventional accelerators and speculated on an early version of a channeling accelerator. In his words "To anyone who has carried out experiments with a large modern accelerator there always comes a moment when he wishes that a powerful spatial compression of his equipment could take place. If only the very large and massive pieces could fit in a small room!" What Hofstadter imagined was a tabletop accelerator he called Miniac. The device would consist of a single crystal driven by an x-ray laser. Channeling would be used to focus the beam. Hofstadter realized the device might be an after-burner to boost a conventional accelerator beam that was already up into the relativistic regime. At the time channeling was a new subject and $\mathrm{x}$-ray lasers were distant dreams.

The limitations of conventional particle accelerators are widely recognized; magnetic fields for circular machines capped at 10 Tesla and RF gradients on the order of $0.5 \mathrm{MeV} / \mathrm{cm}$. With the invention of the laser many explored the possibility of pure laser accelerators. Unfortunately the electric vector for a typical electromagnetic wave points transverse to the direction of propagation and optical frequencies make the construction 
of an electromagnetic cavity outside of the reach of conventional technology.

Nevertheless a number of ingenious ideas for laser acceleration have been put forward including Palmer's comprehensive 1980 study $^{2}$. In the last several years there has been some real progress on this at Brookhaven ${ }^{3}$. A large-scale R\&D program, LEAP, is now underway at Stanford/SLAC ${ }^{4}$.

A different approach has been to accelerate particles using a plasma wave. Some noteworthy discussions of this include articles by Dawson ${ }^{5}$ and his collaborators as well as the comprehensive review by Esarey et al. ${ }^{6}$ All of these approaches use gas plasmas, not a plasma generated in a solid.

In the last years there has been progress on something that might at first glance be considered solid-state acceleration. Groups from Livermore ${ }^{7}$, Michigan $^{8}$, Rutherford $^{9}$ and LULI $^{10}$ have all seen energetic ions and electrons emanating from thin foils struck by extremely powerful picosecond laser pulses. At Livermore they observe "beams" of $10^{13}$ protons downstream of a foil irradiated with a $1000 \mathrm{TW}, 3 * 10^{20} \mathrm{~W} / \mathrm{cm}^{2}$ laser pulse. A beam can be focused by curving the foils. The high-energy ions originate from deposits or contaminants on the downstream side of the foil. The accelerating electrostatic field at the downstream surface is produced by ponderomotively accelerated hot electrons generated by the laser pulse. While this is an interesting process it should probably not be considered a solid-state accelerator since the electrostatic field is outside the solid.

In the late 1990s Helen Edwards' group at Fermilab built a prototype photoinjector at A0 to work on development of the Tesla injector ${ }^{11}$. The accelerator can deliver very large $14 \mathrm{MeV}$ picosecond electron pulses on the order of 10 nanocoulombs or $10^{6} \mathrm{~A} / \mathrm{cm}^{2}$.

The Fermilab A0 photoinjector offered a means to probe in the direction of channeling conditions characteristic of those needed for solid-state acceleration and do observations of channeling under conditions never studied before. The channeling radiation experiment discussed here ${ }^{12}$ was designed to initiate this program.

\section{Plasma Wake Field Acceleration}

In wake field acceleration a laser or electron beam driver creates a moving wave in a plasma. In turn that wave accelerates a charged particle. Figure 1 illustrates a metaphor for the plasma wake field acceleration process. Notice that the surfer in the figure does not have a rope attaching him to the boat as he would if he was a water skier. In this illustration the driver is a powerful motorboat. The particle, here a surfboarder, is pushed along by the wave. Phase stability is important. If the surfer drifts too far down the wave he no longer moves forward. He can accelerate for a while by moving up the wave. The plasma wake field acceleration process is very similar to the metaphor.

The gradient in a plasma is $G \approx 0.96 \sqrt{n_{0}}$ where $n_{0}$ is the electron density (per $\mathrm{cm}^{3}$ ) and $G$ is in $\mathrm{V} / \mathrm{cm}$. For a good electron plasma in a gas the density might reach 
$10^{18} / \mathrm{cm}^{2}$ and produce a gradient of $1 \mathrm{GV} / \mathrm{cm}$. For a solid the density could be 10,000 times higher corresponding to $100 \mathrm{GV} / \mathrm{cm}$. This is a step in the direction Hofstadter was hoping for.

In the last several years SLAC has run a series of increasingly sophisticated wake field acceleration studies ${ }^{13}$. Typically a $30 \mathrm{GeV}$ electron or positron beam impinges on a meter-length Li plasma produced by an ionizing laser. The head of the beam produces a wakefield. That wake field accelerates the tail to $\mathrm{O}(100 \mathrm{MeV})$ depending on conditions. At Fermilab Barov et al. ${ }^{14}$ have produced similar results with the A0 photoinjector.

\section{The Advantages and Challenges of Crystal Acceleration}

The basic solid-state crystal acceleration paradigm is to excite a plasma wake field in a crystal with a density thousands of time higher than a gas plasma. This possibility has been explored in some detail by Chen and Noble ${ }^{15}$. Recent developments in femtosecond laser and electron beam technology make this possibility thinkable. Channeling could be used to reduce energy loss, to focus the particles, and perhaps even cool the beam. But there are big problems. The electron or laser driver beam is so powerful that the crystal may be blown away. In addition there is the classical problem of dechanneling.

To consider a crystal accelerator one must first understand what happens to a crystal exposed to such intense radiation. Chen and Noble ${ }^{16}$ have developed sketches of the process that are useful guides. Initially an electronic plasma is excited by so-called tunnel ionization. The electron plasma frequency is

$$
\omega_{p}=\left(4 \pi n_{0} e^{2} / m_{e}\right)^{1 / 2}
$$

The electronic plasma decays via electronic interband transitions with plasma lifetimes in the femtosecond range. That decay to a hot electron gas excites phonons in the lattice. That can lead to crystal disorder, fracture, or vaporization. The ionic plasma lifetime goes as the square root of the ratio of ionic mass divided by the electron mass so that serious lattice damage may occur within 10 to $100 \mathrm{fs}$. At Livermore ${ }^{17}$ hydrodynamic heating is seen to occur in the 1 to $10 \mathrm{ps}$ range. These times are short but not so short that one can discount the possibility of plasma acceleration.

These comments have to be understood in the context of what is required for acceleration and some other observations of materials exposed to intense laser and particle beams. For a plasma gradient of $1 \mathrm{GeV} / \mathrm{cm}$ the required laser power density is of order $10^{19} \mathrm{~W} / \mathrm{cm}^{3}$. Laser crystal destruction occurs for power densities of order $10^{13} \mathrm{~W} / \mathrm{cm}^{3}$. The lattice is ionized with power fluences of $10^{15}$ to $10^{16} \mathrm{~W} / \mathrm{cm}^{2}$. Charge densities of order $10^{16} \mathrm{e} / \mathrm{cm}^{2}$ are required to get near the acceleration regime. Current densities of $10^{11} \mathrm{~A} / \mathrm{cm}^{2}$ can fracture the crystal. Parenthetically, no evidence of electron beam damage was seen over the course of the A0 channeling radiation experiment. On the other hand several adverse effects were observed in the laser system that powers the 
A0 photoinjector. The laser slab ruptured under continuous operation at $10^{3} \mathrm{~W} / \mathrm{cm}^{3}$ corresponding to $10^{15} \mathrm{~W} / \mathrm{cm}^{3}$ for $10 \mathrm{fs}$. There was lens damage at $10^{9} \mathrm{~W} / \mathrm{cm}^{2}$. In principle the laser might be able to operate to deliver 1 Joule to a 10 micron spot in $1 \mathrm{ps}$ or $10^{18}$ $\mathrm{W} / \mathrm{cm}^{2}$. This is already in the regime where it could be an interesting driver for a plasma accelerator.

How would channeling be affected as a crystal vaporized? I call this process dynamic channeling. As far as I know there is no well-developed theory of dynamic channeling. Andersen ${ }^{18}$ has suggested a treatment based on a screening length that increases when the electrons are removed due to ionization. In the Andersen picture the channeling critical angle as a function of temperature and screening length is

$$
\Psi_{1 / 2}=\frac{\Psi_{L}}{\sqrt{2}} \sqrt{\ln \left(\frac{r_{0}^{2}}{u_{2}^{2} \ln 2}\right)+\ln \left(\frac{\left(\sqrt{3} a_{T F}\right)^{2}+u_{2}^{2} \ln 2}{\left(\sqrt{3} a_{T F}\right)^{2}+r_{0}^{2}}\right)}
$$

where $\Psi_{L}$ is the Lindhard angle. Here $\mathrm{r}_{0}$ is some channel radius and $\mathrm{u}_{2}$ is the rms twodimensional lattice vibration amplitude equal to $0.006 \sqrt{T}$ at high temp for $\mathrm{u}_{2}$ in $\AA$ and $\mathrm{T}$ in ${ }^{\circ} \mathrm{K}$. Removing most of the electrons is equivalent to a large screening length or letting the Thomas-Fermi screening length, $\mathrm{a}_{\mathrm{TF}}$, become large. For practical purposes the screening length reaches its limiting value when $a_{\mathrm{TF}}=\mathrm{r}_{0}$. The behavior of the critical angle is shown in Figure 2 as a function of temperature for two different screening lengths. The critical angle for a fixed screening length does drop as the temperature increases but actually increases for larger screening lengths. The point is that the changes with temperature and screening length are not so large. Channeling would seem to survive until the nuclear centers in the lattice have displacements in the $\AA$ range, that is, until the crystal ceases to be a crystal. It would be useful to have a well-developed treatment of dynamic channeling as a framework for future studies of channeling under conditions similar to those needed for solid-state acceleration. Lacking a concrete theory, the dotted line in Figure 3 suggests how the channeling radiation yield might diminish as the beam intensity increased to the regime where the crystal is vaporized.

\section{The Fermilab A0 Studies}

The Fermilab channeling radiation experiment was undertaken to extend channeling studies toward the solid-state acceleration regime. Channeling radiation was investigated at $\mathrm{A} 0$ in part because earlier experiments had looked at this and in part because it was practical to do a channeling radiation experiment at the high bunch charges at A0.

Only one study of the effect of increasing bunch charge on channeling had been undertaken prior to the Fermilab A0 experiment. This was a channeling radiation study at Darmstadt at $5.4 \mathrm{MeV}$ aimed in part at studying the practicality of channeling radiation for medical applications ${ }^{19}$. In addition there was a single measurement at $\mathrm{Stanford}^{20}$ at 30 $\mathrm{MeV}$. The Stanford measurement was for a different crystal orientation. The Stanford 
value at higher bunch charge was lower that the Darmstadt measurements which appeared to be flat with increasing bunch charge.

As noted earlier the Fermilab photoinjector was able to produce extremely large bunch charges. In addition it was also possible to operate with dark current alone and thereby get a $10^{5}-10^{6}$ reduction in bunch charge so that the experiment was able to cover a wide dynamic range.

In the A0 experiment electrons moving along a crystal axis or plane produced channeling radiation. The electron beam typically had an emittance of $10 \mathrm{~mm} * \mathrm{mrad}$ with a $10 \mathrm{ps}$ long bunch. The beam spot size was characteristically $0.5 \mathrm{~mm}(\sigma)$. A spectrometer magnet swept the beam into a Faraday cup and beam dump. An integrating current transformer was also used to monitor the bunch charge. The channeling radiation showered in a calcium tungstate sheet and produced visible photons that were detected by a photosensitive device.

The design of the crystal goniometer was predicated on the extremely tight requirements on both vacuum and the need for a dust-free environment for the photoinjector and the superconducting cavity. In addition a large diameter, thin silicon crystal was used to eliminate background from the crystal holder due to beam halo. It was mounted with the $<100>$ axis along the beam line. The crystal was aligned by looking at the $\mathrm{x}$-ray signal as a function of the goniometer angles. The experiment consisted of determining the axial and planar yields as a function of bunch charge and dark current.

Because of the extremely high rate it was not possible to count individual particles or photons. Two different detection approaches were used, both employing $\mathrm{CaW}$ screens viewed by either a photomultiplier (AberX lite) or a CCD camera (AberX). The photo-detectors were calibrated by placing them in a variable mono-energetic $\mathrm{X}$-ray beam at the Argonne Advanced Photon Source.

For a twelve decade span of bunch charge Figure 3 shows the channeling radiation yield as $\mathrm{x}$-rays/bunch in a $10 \%$ band. This unit was used to facilitate comparison to the earlier Darmstadt data. The A0 phototube data are represented by open and filled diamonds obtained for axial channeling from Si with the laser on and with dark current, respectively, and for the AberX detector as indicated by the open triangle for planar channeling. The filled circles result from the earlier Darmstadt measurement of axial channeling in a diamond crystal with electrons of $5.4 \mathrm{MeV}$ and the filled triangle from the Stanford measurement of planar channeling in a Si crystal at $30 \mathrm{MeV}$. All points have been scaled to $\mathrm{T}=5.4 \mathrm{MeV}$. Over the 12 decades of the measurements the data trend for the yield per electron is flat.

\section{The Future of High Bunch Charge Channeling Measurements Beyond A0}

Two advances are required to move channeling studies fully into the plasma acceleration regime. One is to increase bunch charge per unit area by factors of $10^{3}$ to $10^{5}$. Part of this can be accomplished by using more focused beams. The second is to use 
pulse lengths in the $10 \mathrm{fs}$ regime. This is challenging but there have recently been significant developments in femtosecond laser technology that could help. Higher beam energies might reduce beam size and perhaps also help for channeling studies. However high energy channeling experiments are different and often harder to arrange than the A0 study.

An example of a potential facility for investigating channeling radiation is E164 at the SLAC $30 \mathrm{GeV}$ FFTB facility ${ }^{21}$. This is being used for continuing plasma acceleration studies. By adding a crystal and a high energy gamma ray detector it might be possible to do a channeling radiation study there along the lines of experiments carried out at Serpukhov ${ }^{22}$ and CERN ${ }^{23}$. The relative beam charge at SLAC is less than at A0 but the beam cross section is substantially smaller so that the bunch charge per unit area would be 500 times larger. The potential reach of SLAC E164 relative to A0 is schematically shown in Figure 3 (schematically because the graph is expressed per bunch while the relevant factor is bunch charge per unit area). The 300 femtosecond pulse length at the facility is a step forward but not all the way to the plasma acceleration regime.

A second possibility is to use the $100 \mathrm{TW}$ laser facilities at Livermore to get extremely high beam currents. A 100 fs laser capable of producing a 50 micron spot with a beam power density of $5 * 10^{18} \mathrm{~W} / \mathrm{cm}^{2}$ is used at Livermore to generate protons by a pseudo solid state acceleration process in the first foil. The proton beam produces $4 \mathrm{eV}$ plasma conditions in a second foil. Could one do channeling studies with this geometry by replacing the second aluminum foil with a crystal? One possibility might be to try Rutherford back scattering although it is not obvious how the backscattering detector could be incorporated in the geometry. Another possibility might be a blocking experiment. Lattice behavior with time could be studied using pump and probe and streak camera techniques. "Available" petawatt lasers could get up into the $10^{14}$ protons/bunch regime.

There have also been recent studies in other fields that suggest different paths to follow for investigations of crystal lattices in dynamic situations. An intriguing example is a study of laser melting at Toronto using sub-picosecond electron diffraction ${ }^{24}$. They use a special 500 fs electron gun to study electron diffraction as a very thin polycrystalline aluminum foil is heated by a laser that is weak by the standards discussed above. The transition from the electronic plasma stage to phonon melting is clearly indicated in the successive Bragg diffraction pictures.

These are three very recent illustrations of potential experimental approaches from a rapidly emerging field studying behavior of solids under dynamic conditions. Many of the possibilities are driven by developments in laser technology. Channeling may have something to offer these studies. Conversely, one might learn interesting things about channeling. And maybe, one may be able to take a step toward solid-state acceleration employing oriented crystals.

\section{Acknowledgements}


The help of J. Freudenberger, S. Fritzler, H. Genz, A. Richter, A. Ushakov, and A. Zilges (Darmstadt), J. P. F. Sellschop (Witwatersrand), H. Edwards, W. Muranyi, J. Santucci (Fermilab), D. Haeffner, P. Lee, A. Mashayekhi, A. McPherson (Argonne), W. Hartung (Michigan State), R. Noble (Stanford), J. Carneiro (DESY), M. Fitch (Johns Hopkins), and N. Barov (NIU) is gratefully acknowledged.

*Operated by Universities Research Association, Inc. under contract No. DEAC02-76CHO3000 with the United States Department of Energy

\section{Figure captions}

Figure 1 Wake field surfer metaphor for plasma wakefield acceleration (courtesy S. Carrigan).

Figure 2 Axial critical angle for silicon as a function of temperature for two different screening lengths.

Figure 3 Channeling radiation yield (x-rays/bunch in a 10\% band) over a twelvedecade span of bunch charge. The present data are represented for the AberX-Lite detector by open and filled diamonds obtained for axial channeling from $\mathrm{Si}$ with the laser on and with dark current, respectively, and for the AberX detector as indicated by the open triangle for planar channeling. The filled circles result from an earlier measurement of axial channeling in a diamond crystal with electrons of $5.4 \mathrm{MeV}$ and the filled triangle from planar channeling in a Si crystal at $30 \mathrm{MeV}$. All points have been scaled to $\mathrm{T}=5.4$ $\mathrm{MeV}$. 


\section{Figures}

Figure 1

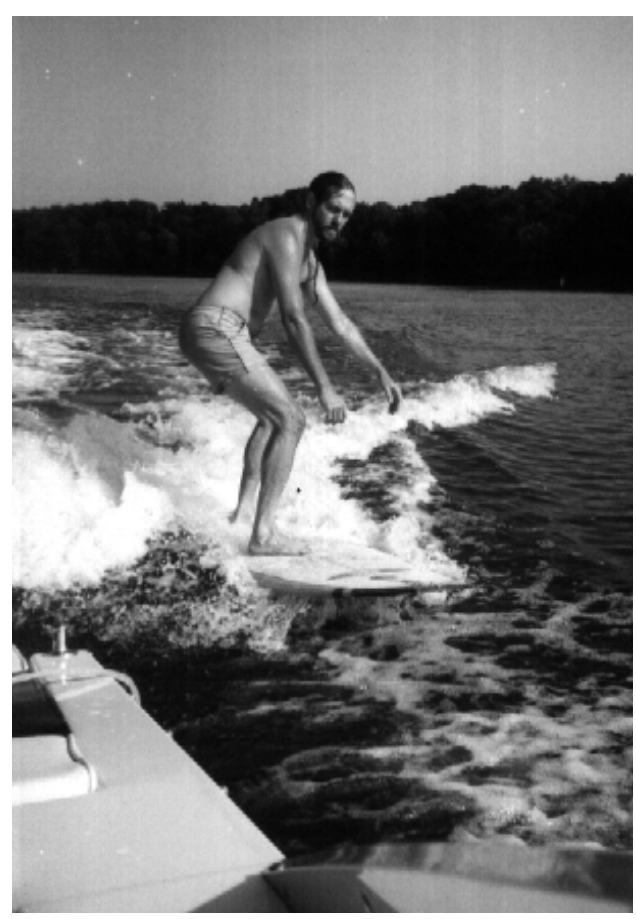


Figure 2

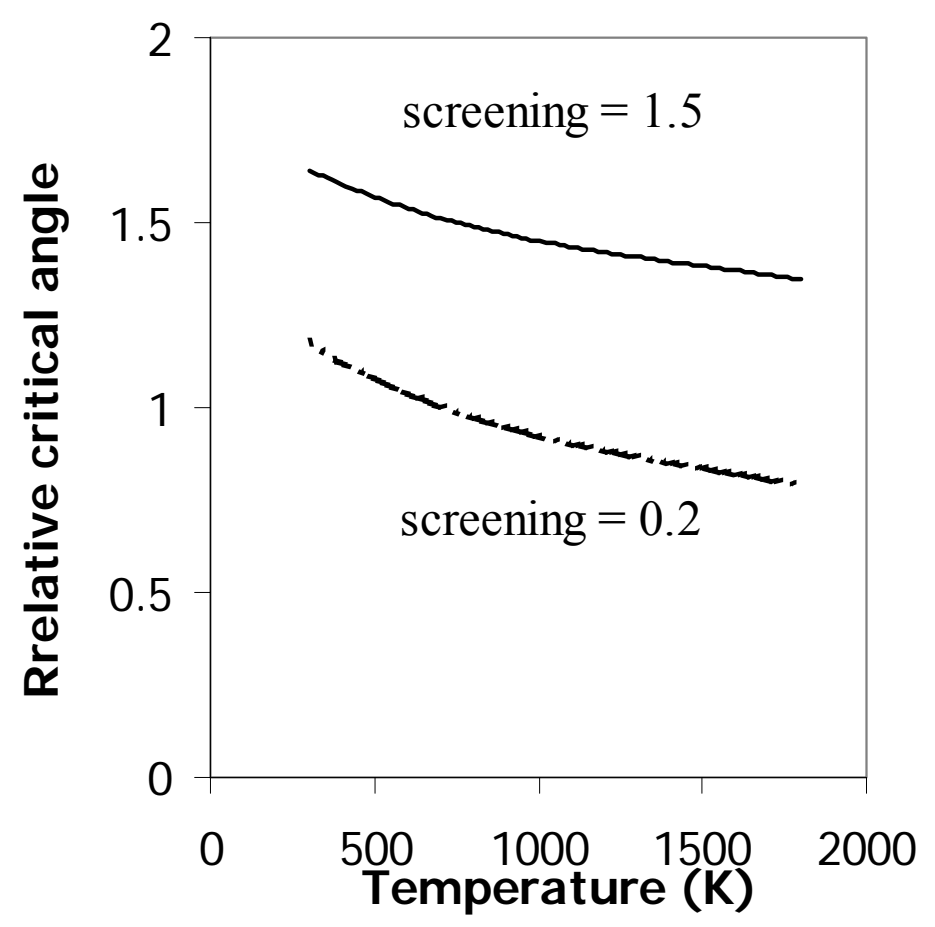

Figure 3

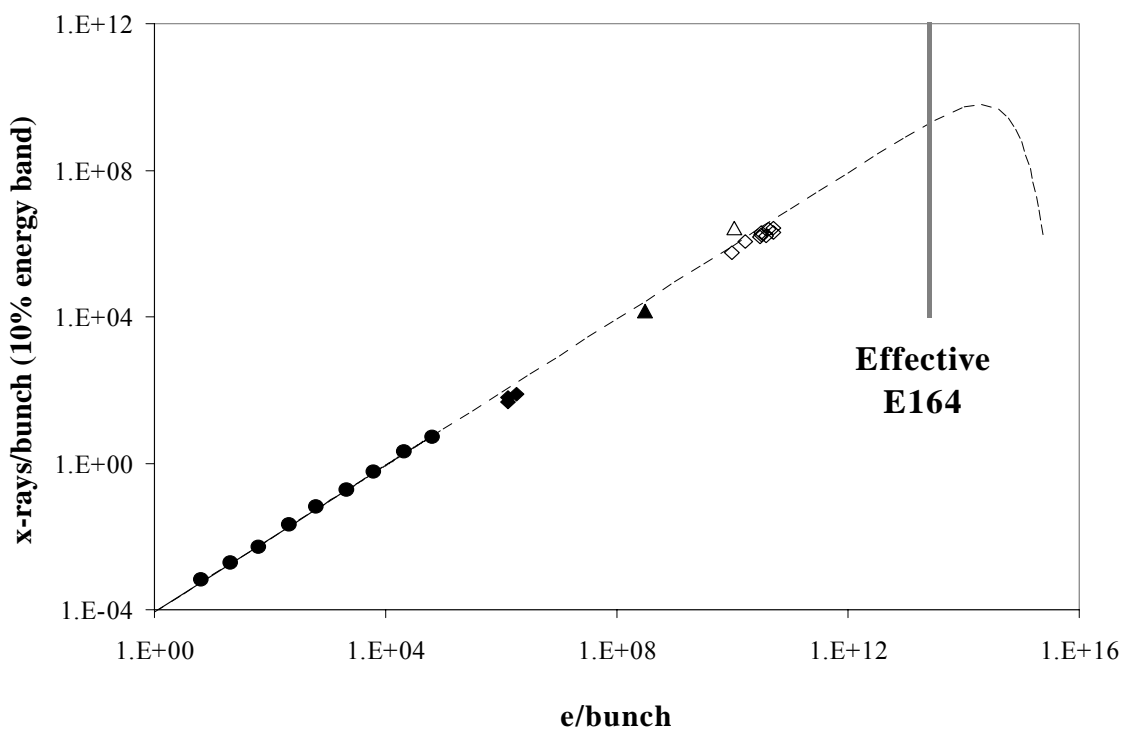


${ }^{1}$ R. Hofstadter, Stanford HEPL Report 560 (1968).

${ }^{2}$ R. B. Palmer, Particle Accelerators 11, 81 (1980).

${ }^{3}$ W. D. Kimura et al., PRL 92, 054801 (2004).

${ }^{4}$ C. D. Barnes, E. R. Colby, and T. Plettner, p. 294 in Advanced Accelerator Concepts: Tenth Workshop, eds. C. E. Clayton and P. Muggli, Amer. Inst. of Physics Press CP647, New York (2002).

${ }^{5}$ T. Tajima and J. M. Dawson, Phys. Rev. Lett. 43, 267 (1979).

${ }^{6}$ E. Esarey, et al., IEEE Trans. On Plasma Sci. 24, 252 (1996).

${ }^{7}$ R. A. Snavely et al., Phys. Rev. Lett. 852945 (2000).

${ }^{8}$ K. Nemoto, et al., Applied Physics Lett. 78, 595 (2001).

${ }^{9}$ M. Zepf, et al., Phys. Rev. Lett. 90, 064801 (2003).

${ }^{10}$ M. Roth, et. al., Phys. Rev. Special Topics - Accelerators and Beams 5, 061301 (2002).

11 J. P. Carneiro et al., FERMILAB-Conf-99/271, 1999.

${ }^{12}$ R. A. Carrigan, Jr. et al., Phys. Rev. A68, 062901 (2003).

${ }^{13}$ M. J. Hogan, et al. Phys. Plasmas 7, 2241 (2000).

${ }^{14}$ N. Barov et al. Particle Accelerator Conference Proceedings (2001), Fermilab-Conf-01/365.

${ }^{15}$ P. Chen and R. J. Noble, p. 273 in Advanced Accelerator Concepts, eds. S. Chattopadhyay, J. McCullough, and P. Dahl, Amer. Inst. of Physics Press C398, New York (1997).

${ }^{16}$ P. Chen and R. J. Noble , p. 517 in Relativistic Channeling, eds. R. A. Carrigan, Jr. and J. A. Ellison (Plenum, 1987).

${ }^{17}$ P. K. Patel, et al., Phys. Rev. Lett. 91, 125004 (2003).

${ }^{18} \mathrm{~J}$. U. Andersen, private communication.

${ }^{19}$ H. Genz, H.-D. et al., App. Phys. Lett. 57, 2956 (1990). W. Lotz, et al. Nucl. Instr. and Meth. B48, 256 (1990).

${ }^{20}$ C. K. Gary, et al. Nucl. Instr. and Meth. B51, 458 (1990). C. K. Gary, et al., Phys. Rev. B42, 7 (1990).

${ }^{21}$ C. D. Barnes et al., Proc. 2003 Particle Acc. Conf. 1530 (2003).

22 N. A. Filatova, et al., Phys. Rev. Lett. 48, 488 (1982).

${ }^{23}$ K. Kirsebom, et al., Nucl. Instr. and Meth. 119, 79 (1996).

${ }^{24}$ B. J. Siwick, et al., Science 302, 1382 (2003). 